\title{
Manejo actual de la microtia: redefinición anátomo-quirúrgica*
}

\author{
Drs. JUAN PABLO SOROLLA P. ${ }^{1}$, DOUGLAS ARBULO L. ${ }^{1}$, MIGUEL OBAID G. ${ }^{1}$, \\ Int. CARLA IBARRA M. ${ }^{2}$, Drs. MONTSERRAT FONTBONA T. ${ }^{1}$, \\ RODRIGO CABELLO P. ${ }^{1}$, PAMELA WISNIA C. ${ }^{1}$, ÁNGELA BAUTISTA S. ${ }^{1}$
}

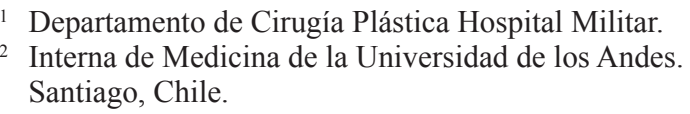

\section{Abstract \\ Management of microtia based on a redefinition of its anatomical-surgical classification}

Background: Incomplete development and growth of the pinna can lead to a small or deformed pinna, called microtia. Aim: To report and evaluate the effectiveness of a surgical technique previously described for the treatment of microtia, based on a new anatomical-surgical classification. Materials and Methods: Retrospective study of patients treated at two plastic surgery departments from January 2008 to December 2010. We recruited a total of 15 patients aged 9 to 25 years. Thirteen $(87 \%)$ had unilateral and $2(13 \%)$ bilateral microtia. All patients underwent the surgical reconstructive technique described by Firmin. Results: Among patients with unilateral microtia, seven belonged to Firmin type I, five to type II and one to type IIIa. Both patients with bilateral microtia, were classified as type IIIlb. In all cases with unilateral microtia, a good initial projection of the cartilage frame was achieved during the first surgical procedure. Six patients were subjected to a second operation. During 18 months follow up, four patients (16\%) had complications. Conclusions: This surgical technique that is based on an anatomical surgical classification, achieves favorable and satisfactory results.

Key words: Microtia, reconstruction, Firmin classification.

\section{Resumen}

Objetivos: Reportar y evaluar la efectividad de una técnica quirúrgica, previamente descrita para el tratamiento de microtias, basado en un nuevo concepto de clasificación anátomo-quirúrgica. Material y Método: Estudio retrospectivo de pacientes tratados en el Hospital Militar y Clínica Alemana de Santiago en el Servicio de Cirugía Plástica desde enero de 2008 hasta diciembre de 2010. Se reclutaron a un total de 15 pacientes, $13(87 \%)$ de los cuales eran unilaterales y $2(13 \%)$ bilaterales. A todos los pacientes se les realizó la reconstrucción mediante técnica quirúrgica de Firmin. Resultados: Se operó a 15 pacientes de rango

*Recibido el 2 de febrero de 2012 y aceptado para publicación el 10 de junio de 2012.

Los autores no refieren conflictos de interés.

Correspondencia: Juan Pablo Sorolla P.

Agustín de Negri 5241, Santiago, Chile.

ipsorolla@gmail.com 
de edad entre 9 y 25 años, con una mediana de 11 años. De los casos unilaterales; 7 correspondían a tipo I de Firmin, 5 casos a tipo II Firmin y un caso tipo IIIa. De los casos bilaterales, ambos tenían microtia tipo IIIb. Se logró en la totalidad de los casos unilaterales una buena proyección inicial del marco cartilaginoso durante el primer tiempo quirúrgico. Seis casos fueron sometidos a un segundo tiempo quirúrgico. Durante el seguimiento mínimo de 18 meses, se observaron complicaciones en 4 (16\%) pacientes. Conclusiones: Se comprueba que mediante una técnica basada en la clasificación anátomo-quirúrgica, los resultados a corto plazo fueron favorables y satisfactorios.

Palabras clave: Microtia, reconstrucción, Firmin.

\section{Introducción}

Los pabellones auriculares son componentes estéticos importantes para el humano. Para que el cirujano pueda corregir la deformidad, éste debe poseer un vasto conocimiento de la anatomía auricular y sus variaciones encontradas en la deformidad congénita. La prevalencia de las malformaciones auriculares tales como microtia y anotia varían entre 0,8 y 2,4 por 10.000 nacimientos, con una menor prevalencia en europeos y negros en comparación con hispánicos y asiáticos ${ }^{1}$. Se produce entre la quinta y sexta semana de gestación y resulta de componentes deficientes o supernumerarios. Su etiología es multifactorial e incluye factores genéticos, teratogénicos y anormalidades vasculares ${ }^{2}$. En los casos unilaterales es más preponderante en el lado derecho y en hombres ${ }^{2}$. Esta malformación se asocia a otros defectos congénitos como paladar hendido, malformaciones cardíacas y renales, anoftalmia, microftalmia, entre otras ${ }^{2}$. Existen numerosas clasificaciones de los diferentes tipos de microtia, previo a Firmin ninguna de ellas se relacionaba en forma anátomo-quirúrgica. De esta manera se ha simplificado la reconstrucción del marco auricular y el tipo de incisión a utilizar.

La reconstrucción de la microtia es uno de los procedimientos más desafiantes en la cirugía plástica $^{3}$, tanto por su arquitectura tridimensional como por múltiples reparos anatómicos ${ }^{4}$. Para esto se pueden utilizar soportes de cartílago costal autólogo que tienen mejor pronóstico a largo plazo o implantes protésicos ${ }^{5}$.

Una reconstrucción exitosa incluye una adecuada replicación de los reparos anatómicos, tamaño preciso, buena localización y proyección ${ }^{4}$. Esto resulta de una adecuada preparación del soporte cartilaginoso tridimensional y una estrategia apropiada para adaptar el remanente de piel al molde cartilaginoso ${ }^{6}$. Por ello la elección del tipo de bolsillo de piel resulta de gran importancia para lograr un resultado satisfactorio ${ }^{6}$.

Nuestro trabajo busca reportar y evaluar la efectividad de una nueva técnica quirúrgica basada en la clasificación anátomo-quirúrgica según Firmin. Esta se compone en dos etapas:
Primer Tiempo: Creación del molde según clasificación e inserción bajo la piel.

Segundo Tiempo: Elevación de la reconstrucción auricular y recreación del surco retroauricular.

\section{Material y Método}

\section{Diseño}

Estudio retrospectivo de pacientes tratados en el Hospital Militar y Clínica Alemana de Santiago en el Servicio de Cirugía Plástica desde enero de 2008 hasta diciembre de 2010. Se reclutaron un total de 15 pacientes, $13(87 \%)$ de los cuales eran unilaterales y $2(13 \%)$ bilaterales, correspondiendo a 10 hombres y 5 mujeres. Todos los pacientes resueltos mediante técnica quirúrgica de Firmin por un único cirujano, registrando datos demográficos, protocolo operatorio, complicaciones precoces y tardías, seguimiento clínico y fotográfico mínimo de 18 meses y valoración de la satisfacción del paciente mediante la aplicación de escala valórica de Strasser con cuatro observadores (padres, enfermera y Dra Firmin) ${ }^{7}$.

Todos los pacientes que ingresaron al estudio, lo hicieron con el previo consentimiento informado de sus padres o por ellos mismos los mayores de 18 años.

Los criterios de inclusión fueron: paciente con microtia uni o bilateral y mayor o igual a 9 años. Y criterio de exclusión: índice peso/talla bajo para su edad. (-2 DE)

Las variables que se evaluaron correspondieron a: edad, sexo, microtia uni o bilateral, número de intervenciones, número de complicaciones y escala valórica de Strasser.

Todos los datos fueron tabulados y analizados en Microsoft Office Excel 2007.

\section{Procedimiento}

En la totalidad de los pacientes se realizó la reconstrucción mediante una técnica basada en la clasificación anátomo-quirúrgica.

En los casos de microtia unilateral, el molde se obtuvo de la oreja contralateral sana, con una placa de rayos marcando los reparos anatómicos; se invierte y se hace unos milímetros más pequeño para acomodarlo al grosor de la piel que lo va a recubrir. 
En casos de anotia o microtia bilateral el molde se obtuvo del padre o hermano.

Para la estimación ideal de la ubicación del marco cartilaginoso, se debe tener en cuenta una serie de parámetros que se ilustran en la Figura 1.

En pabellón y bajo anestesia general se obtuvo los cartílagos costales de la $6^{\mathrm{a}}$ a $9^{\mathrm{a}}$ ipsilateral, a través de una incisión oblicua de $4-5 \mathrm{~cm}$.

Una vez extraído el cartílago, éste se voltea para construir y dibujar el molde basado en la oreja contralateral.

Lo primero que se realizó fue esculpir la base, profundizando el área de la escafa y la fosa triangular, para luego añadir las diferentes piezas de hélix, antihélix, y el complejo trago-antitrago para formar la arquitectura tridimensional dependiendo de la clasificación. Se fijaron a la base con sutura de acero inoxidable de doble aguja, recta y atraumática 5-0 (Figura 2).

Un segmento de cartílago se guardó en el tejido subcutáneo en el área del abordaje de los cartílagos costales, en preparación para la proyección de la concha auricular durante el segundo tiempo quirúrgico.
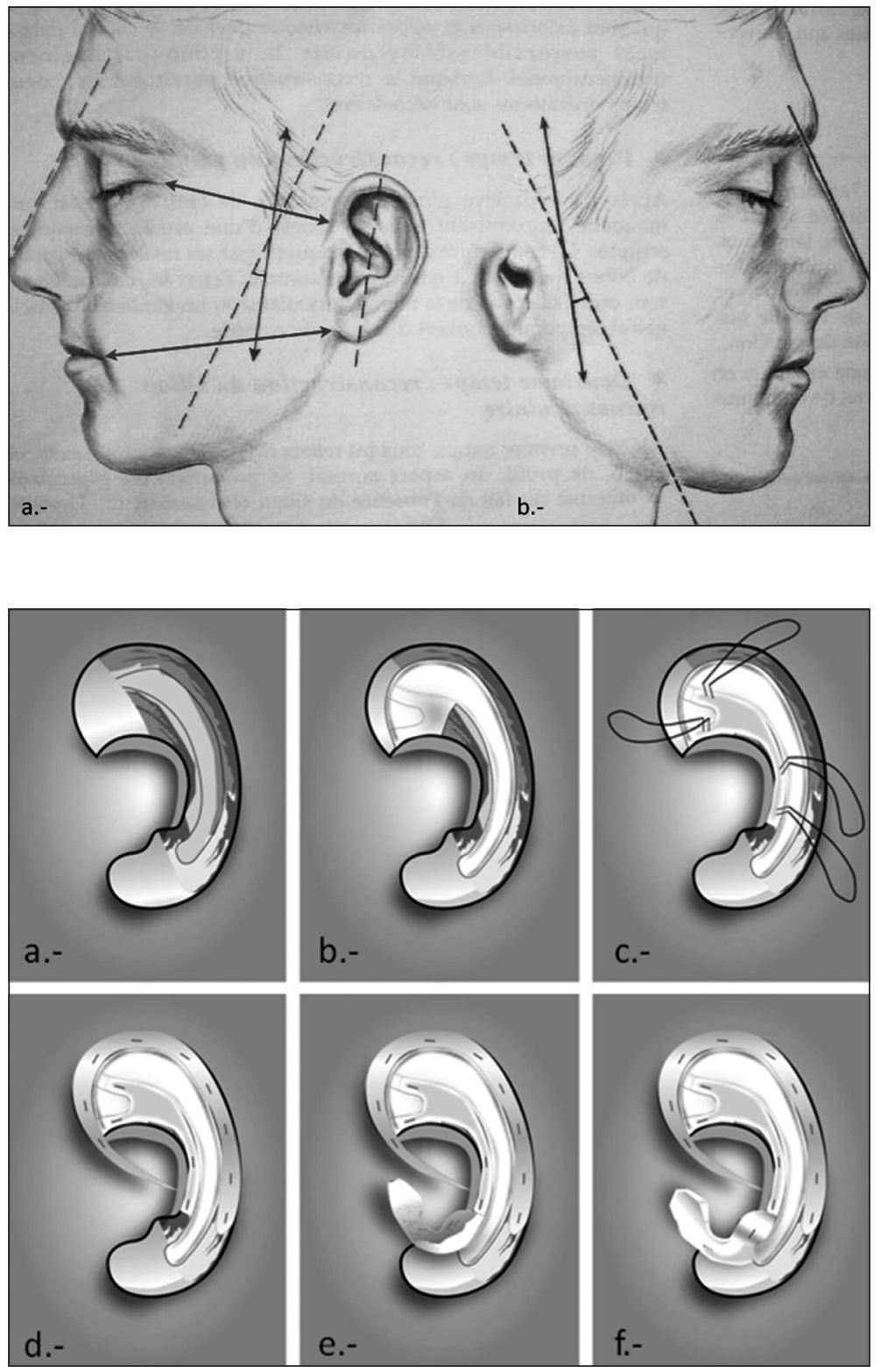

Figura 1. Orientación para la colocación del marco cartilaginoso: a) Visión lateral oreja sana: Se traza una línea paralela al dorso de la nariz y se intersecta con otra línea en un ángulo de $15^{\circ}$. Luego se mide la distancia entre el canto externo del ojo y el origen del hélix y la distancia entre la comisura de la boca y el lóbulo; b) Visión lateral de la deformación auricular: Las mediciones anteriores se utilizan sobre el lado afectado para estimar la colocación ideal del molde cartilaginoso. 
La elección del tipo de bolsillo se decidió teniendo en cuenta las distintas formas de microtia. Para ello se categorizó según los tres tipos de Firmin ${ }^{6}$.

\section{Tipo 1}

Este tipo de incisión permite la transposición de dos colgajos tipo z-plastía reubicando el lóbulo. Técnica descrita por Nagata sin considerar su pedículo (Figura 3).

\section{Tipo II}

Incisión transfixiante que involucra piel y fibrocartílago hasta la zona del proceso mastoideo. Permite que la parte inferior del molde forme el lóbulo (Figura 4).

\section{Tipo III}

Incisión de piel. Se utiliza sólo en dos ocasiones.

- Tipo IIIa: Oreja de tamaño normal pero con fibrocartílago deforme en su tercio superior. Se retira el fibrocartílago y se coloca el molde bajo el bolsillo de piel. Ya el surco retroauricular, la concha y el lóbulo estaban presentes (Figura 5).

- Tipo IIIb: Se utiliza cuando no existe fibrocartílago o en caso de anotia. La incisión se efectúa donde le acomode al cirujano. Se realiza el bolsillo y se introduce el molde cartilaginoso (Figura 6).
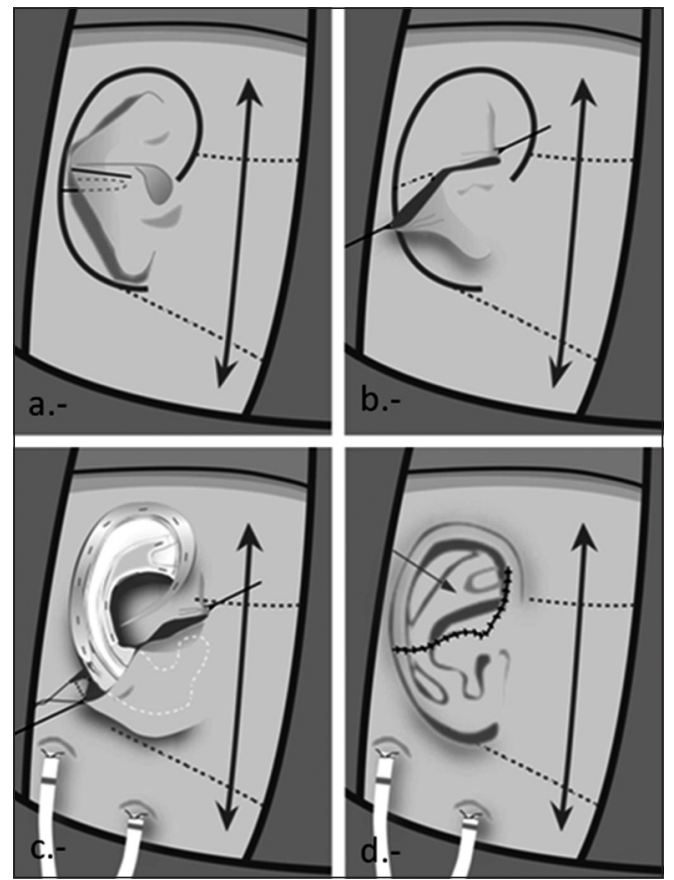

Figura 4. Tipo II de Firmin: a) Incisión transfixiante que involucra piel y fibrocartílago; b y c) Creación del bolsillo e introducción de la porción inferior del molde cartilaginoso para la creación del lóbulo; d) Resultado final.
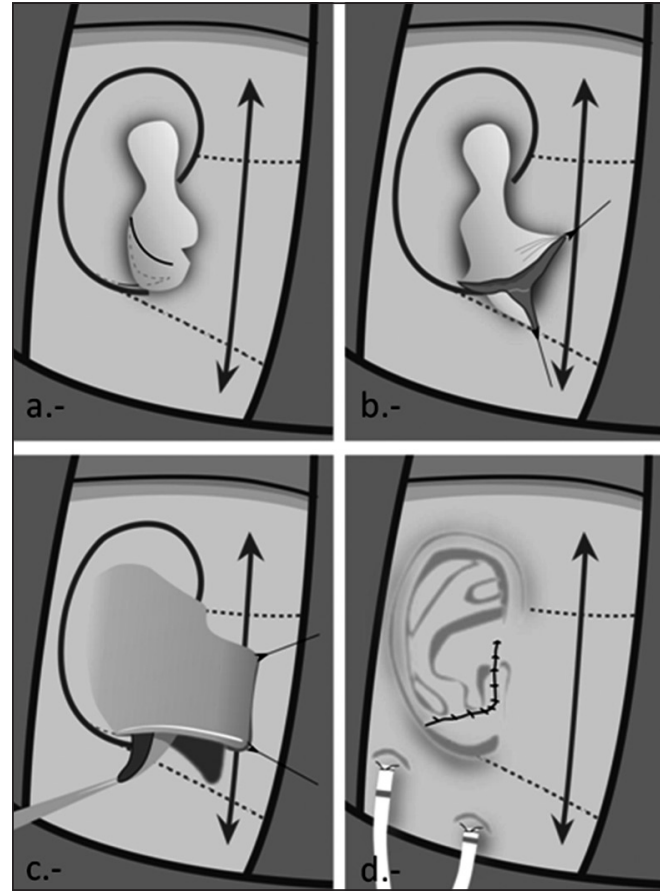

Figura 3. Tipo I de Firmin: a) Incisión tipo Zplastía; b y c) Elevación e intercambio de colgajos; d) Resultado final.
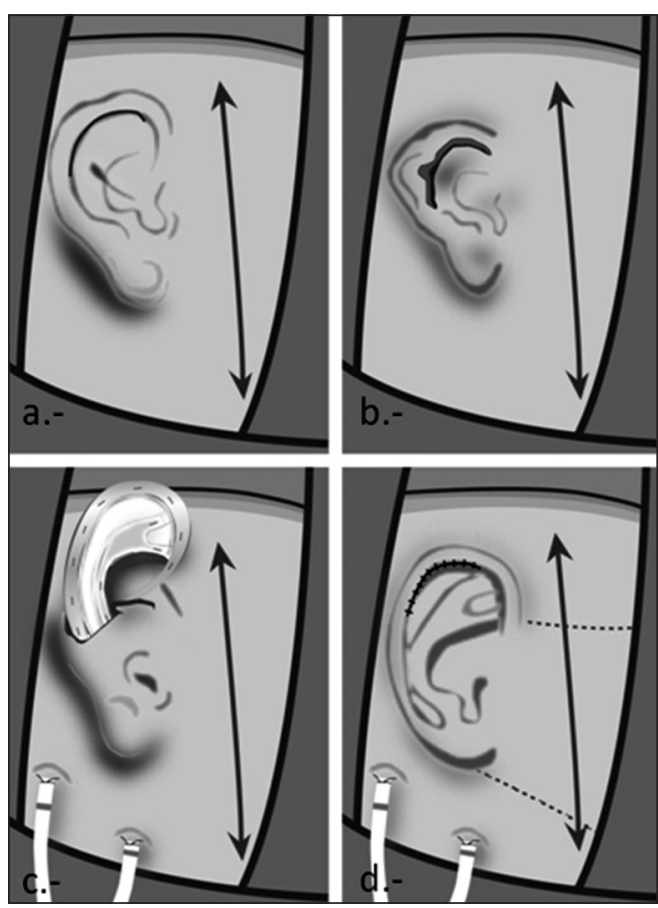

Figura 5. Tipo IIIa de Firmin: a y b) Incisión, remoción del fibrocartílago deforme y creación de bolsillo de piel; c) Introducción del molde cartilaginoso; d) Resultado final. 


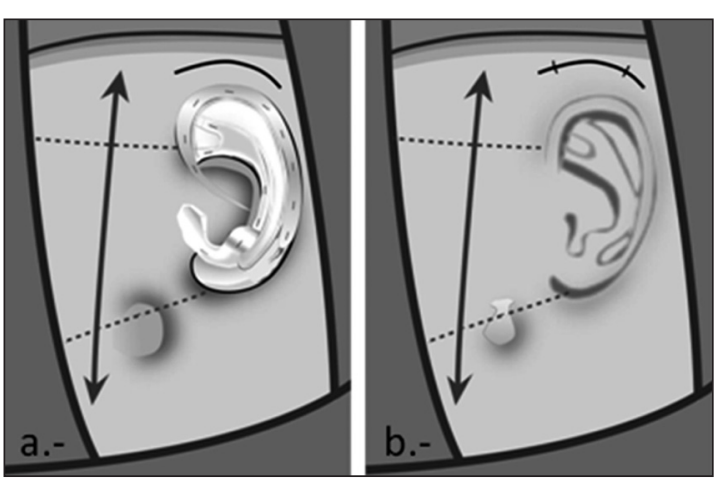

Figura 6. Tipo IIIb de Firmin: a) Incisión y creación de bolsillo de piel; b) Introducción del molde cartilaginoso. Resultado final.

Dos drenajes Blake ${ }^{\circledR}$ de polietileno, multifenestrado de 10 french, mantuvieron a presión negativa el colgajo de piel sobre el nuevo marco cartilaginoso, estos se retiraron durante el $3^{\text {er }} \mathrm{o} 4^{\text {to }}$ día post operatorio. La oreja reconstruida se recubre 8 días con Bactigras ${ }^{\circledR}$ y abundante gasa flu.

Los pacientes que fueron sometidos a un segundo tiempo, este se realizó al menos seis meses después de la primera intervención. La pieza de cartílago guardada en la pared del tórax durante la primera etapa, debe ser preparada antes de ser utilizada para la proyección de la concha auricular.

Un colgajo de fascia temporal con vascularización axial desde la arteria temporal superficial, va a cubrir la superficie posterior del molde, como también la pared posterior reconstruida de la concha. Después de realizar un bolsillo, el vértice posterior de la piel se moviliza hacia arriba y adelante, hacia la profundidad de los surcos ${ }^{6}$.

El defecto de piel en el área de la mastoides y la parte posterior del molde, se cubre con injerto de piel dermo-epidérmico que se obtiene de la región temporal ipsilateral ${ }^{4}$.

\section{Resultados}

Durante el período descrito se trataron 15 pacientes, 13 de los cuales eran unilaterales $(87 \%)$ y $2(13 \%)$ bilaterales, correspondiendo a 10 hombres y 5 mujeres. De los 13 casos unilaterales, hubo predominio del lado derecho con 8 pacientes $(53 \%)$ y 5 izquierdas (33\%). Según el tipo de microtia, 7 casos correspondían a tipo I de Firmin, 5 casos correspondían a tipo II de Firmin y 1 caso a tipo IIIa. De los casos bilaterales ambos tenían microtia tipo IIIb.

Se ilustran reconstrucciones de microtia tipo I y II según Firmin, con marcos tipo I y III en las Figuras 7 y 8 .

Con relación a la etiología de los casos unilaterales, 11 correspondían a microtias aisladas, 1 caso con microsomía facial, 1 caso catalogado como Síndrome de Goldenhar y 2 casos asociados a parálisis facial leve.

El rango de edad de los pacientes operados fue de 9 a 25 años, con una mediana de 11 años.

En relación al número de intervenciones realizadas, se logró en la totalidad de los casos unilaterales una buena proyección inicial del marco cartilaginoso durante el primer tiempo quirúrgico. Seis casos fueron sometidos a un segundo tiempo quirúrgico, donde en tres se utilizó un colgajo de fascia temporal y los tres restantes colgajo de fascia mastoidea.

Durante el seguimiento mínimo de 18 meses, se observaron complicaciones en $4(16 \%)$ pacientes que correspondieron a exposición de hélix de tercio superior (Figura 9), falta de definición de contorno auricular, dehiscencia de sutura de zona dadora y asimetría costal. Por último, la totalidad de los pacientes intervenidos obtuvieron un resultado bueno según la escala de Strasser (Puntaje total entre 1 a 4).

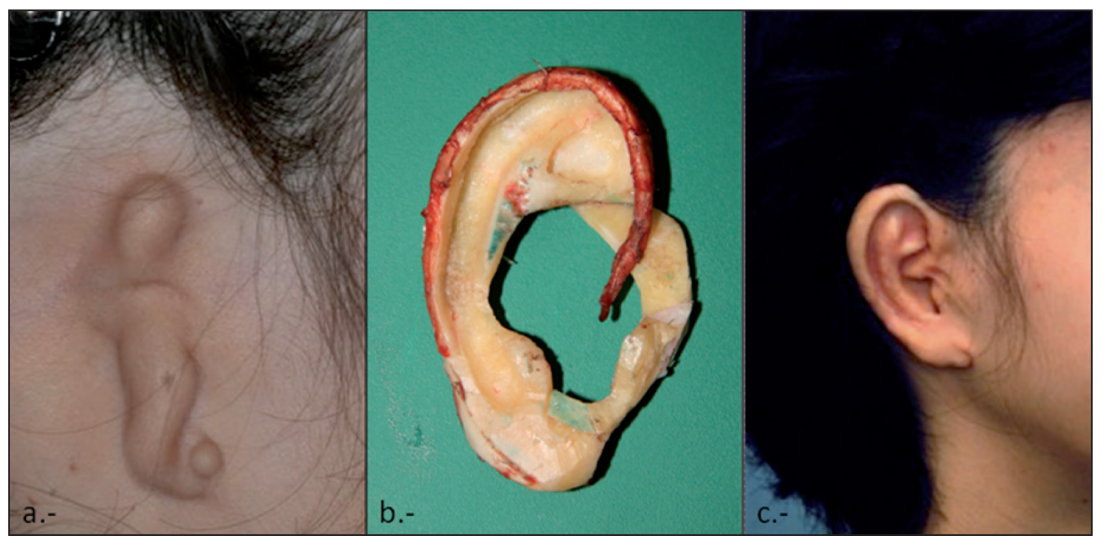

Figura 7. Ejemplo clínico Microtia Tipo I de Firmin (incisión): a) Visión preoperatoria de paciente femenino de 14 años con microtia unilateral izquierda Tipo I según clasificación de Firmin; b) Visión anterior de marco tipo I. c) Resultado final en el postoperatorio de 6 meses. 


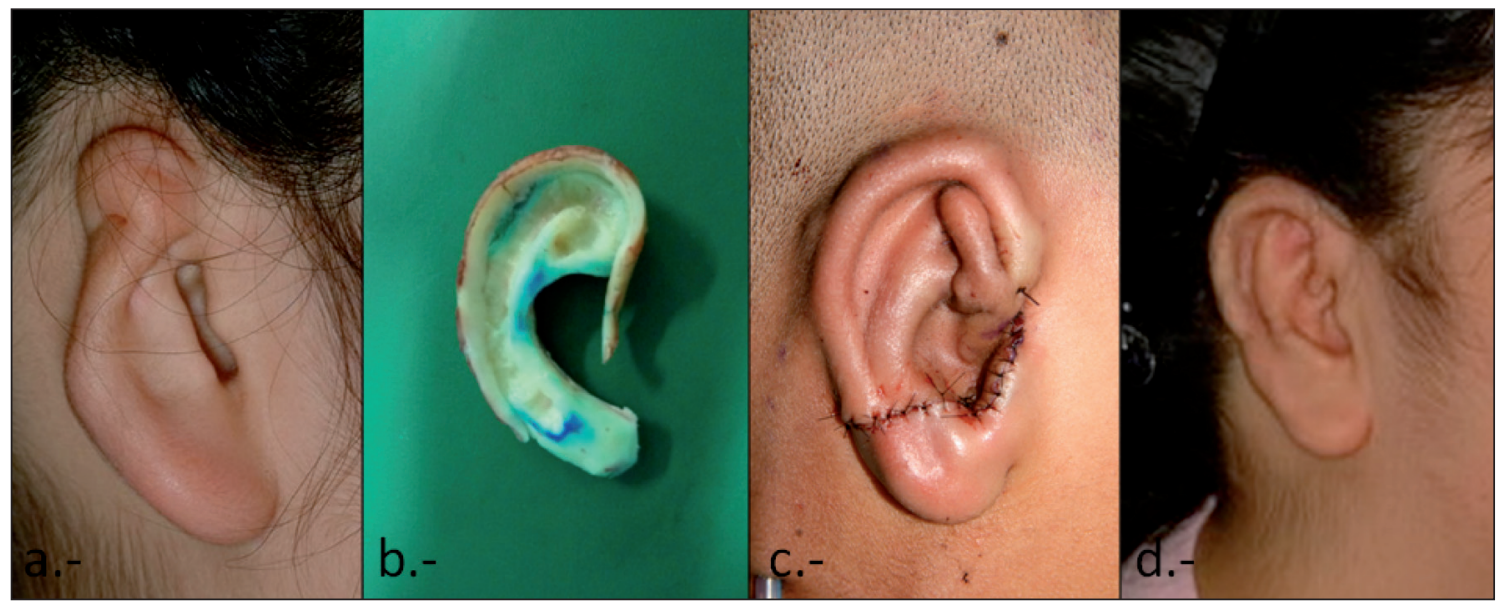

Figura 8. Ejemplo clínico Microtia Tipo II de Firmin (incisión): a) Visión preoperatoria de paciente femenino de 9 años con microtia unilateral izquierda Tipo II según clasificación de Firmin; b) Visión anterior de marco tipo III; c) Resultado final en el postoperatorio inmediato; d) Resultado final en el postoperatorio luego de 6 meses.

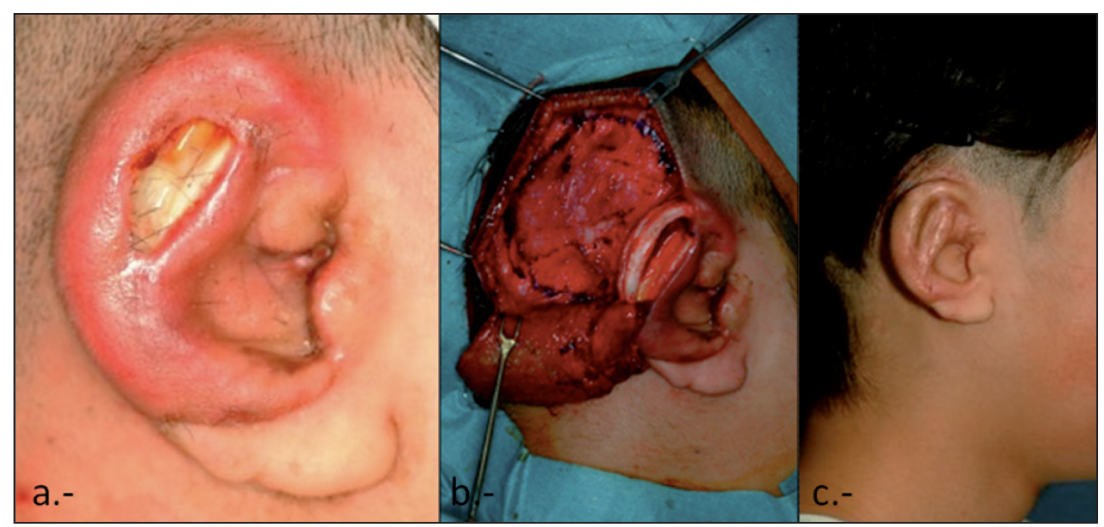

Figura 9. Ejemplo clínico de complicación postoperatoria: a) Paciente masculino de 9 años sufre exposición de hélix de tercio superior luego de ser intervenido por microtia izquierda, con incisión Tipo II según clasificación de Firmin; b) Se decide cubrir zona de defecto con colgajo de fascia temporal sin incidentes durante la intervención quirúrgica; c) Resultado final luego de 1 año de la corrección.

\section{Discusión}

Diferentes técnicas quirúrgicas se han diseñado hasta el momento. La reconstrucción auricular varía ampliamente en su planificación, estadio, técnica quirúrgica, creación del tipo de molde cartilaginoso e incisión dependiendo del tipo de deformidad.

Numerosas modificaciones a la técnica quirúrgica se han realizado en los últimos 20 años, las más aceptadas últimamente tanto por su calidad y reproductibilidad han sido las de Brent y Nagata ${ }^{8}$. Estos dos últimos autores han clasificado los remanentes auriculares en microtias clásicas o atípicas con su abordaje respectivo. Por otra parte, Firmin creó una clasificación quirúrgica la cual basada en los remanentes anatómicos residuales, logra un algoritmo quirúrgico de aproximación para el tipo de marco a reconstruir y la incisión a utilizar.

Firmin por su parte ha logrado masificar su técnica haciéndola reproducible a través de Work Shops basado en videos demostrativos y material para esculpir los diferentes tipos de marcos auriculares.

En nuestra experiencia, la utilización de cartílago autólogo ha demostrado ser la mejor alternativa terapéutica para estos pacientes. A pesar de ello los materiales protésicos deben ser considerados en pacientes con alteraciones en los tejidos locales debidos a intervención quirúrgica previa, irradiación, cáncer, etc ${ }^{8}$.

En cuanto a las limitaciones del estudio, no se realizó un trabajo comparativo entre las distintas técnicas quirúrgicas por un mismo cirujano. El número de pacientes recopilados no parece ser suficiente para emitir resultados concluyentes.

En conclusión, mediante una técnica basada en la clasificación anátomo-quirúrgica, los resultados a corto plazo fueron favorables y satisfactorios. 


\section{Referencias}

1. Porter C. Tan ST. Congenital auricular anomalies: topographic anatomy, embryology, classification, and treatment strategies. Plast Reconstr Surg. 2005;115: 1701-12.

2. Beahm EK, Walton RU Auricular reconstruction for microtia: part I. Anatomy, embryology, and clinical evaluation. Plast Reconstr Surg. 2002; 109:2473-82.

3. Bauer, B. Reconstruction of microtia. Plast Reconstr Surg. 2009;124Suppl:14e-26e.

4. Zhang Q, Zhang R, Xu H, Jin P, Cao Auricular reconstruction for microtia: personal 6-year experience based on 350 microtia ear reconstructions in China. Plast
Reconstr Surg. 2009;12:849-58

5. Thorne CH. Brecht LE, Bradley JA, Levine JA, Hammerschlag 1 , Longaker MT. Auricular reconstruction: indications for autogenous and prosthetic techniques. Plast Reconstr Surg. 2001;107:1241-52.

6. Firmin F. State-of-the-art autogenous ear reconstruction in cases of microtia. Adv Otorhinolaryngol. (Basel, Karger) 2010;68:25-52.

7. Strasser EJ. Application of an Objective Grading System for the Evaluation of Cosmetic Surgical Results. Plast Reconstr Surgery 1999;104:2282-5.

8. Walton RU, Beahm EK. Auricular reconstruction for microtia: Part II. Surgical techniques. Plast Reconstr Surg. 2002;110:234-49. 